The purpose of this Letters section is to provide rapid dissemination of important new results in the fields regularly covered by Physics of Plasmas. Results of extended research should not be presented as a series of letters in place of comprehensive articles. Letters cannot exceed three printed pages in length, including space allowed for title, figures, tables, references and an abstract limited to about 100 words. There is a three-month time limit, from date of receipt to acceptance, for processing Letter manuscripts. Authors must also submit a brief statement justifying rapid publication in the Letters section.

\title{
Sawteeth and energy confinement in the Madison Symmetric Torus reversed-field pinch
}

\author{
B. E. Chapman,, a) A. F. Almagri, M. Cekic, D. J. Den Hartog, S. C. Prager, and J. S. Sarff \\ University of Wisconsin, Department of Physics, 1150 University Avenue, Madison, Wisconsin 53706
}

(Received 23 October 1995; accepted 6 December 1995)

Most Madison Symmetric Torus (MST) [Fusion Technol. 19, 131 (1991)] reversed-field pinch
discharges exhibit sawtooth oscillations with a period of 2-5 ms, corresponding to
magnetohydrodynamic (MHD) instability and increased transport. However, in discharges where the
plasma-facing wall has been boronized, the plasma resistivity is reduced, and sawteeth are often
suppressed for periods up to $20 \mathrm{~ms}$. The energy confinement time during these sawtooth-free periods
is triple the normal value, corresponding to a higher plasma temperature and lower Ohmic input
power. In addition, the steady growth of the dominant magnetic fluctuations normally observed
between sawtooth crashes is absent. () 1996 American Institute of Physics. [S1070-664X(96)02703-5]

Sawtooth oscillations have been observed in several reversed-field pinch (RFP) experiments. ${ }^{1-3}$ Similar to the case in tokamaks, the RFP sawtooth crash coincides with a sudden transfer of energy from the core plasma to the edge and is interpreted as magnetohydrodynamic (MHD) relaxation. During the slow sawtooth rise phase in the RFP, the $\mu$ profile, where $\mu(r)=\mu_{0} \mathbf{J} \cdot \mathbf{B} / B^{2}$, peaks as a result of resistive diffusion and the peaked parallel equilibrium inductive electric field. The amplitudes of the dominant poloidal mode number $m=1$ magnetic fluctuations (or modes), resonant in the plasma core, gradually increase during this phase. The faster sawtooth crash phase results in the redistribution of parallel current, flattening $\mu(r)$. The crash is a discrete dynamo event, during which edge current is driven, and toroidal flux is generated. Sawteeth degrade confinement in two ways. First, the growth of magnetic fluctuations increases transport. Second, plasma-wall interaction, most prominent in the crash phase, injects radiative impurities into the plasma. Hence, control of sawtooth oscillations and understanding of their origin is essential.

In the Madison Symmetric Torus (MST) RFP, ${ }^{4}$ sawteeth with periods of several ms have, until recently, accompanied nearly all discharges. ${ }^{5}$ In this paper we report the production of discharges, following boronization ${ }^{6}$ of the plasma-facing wall, in which sawteeth are absent for extended periods (up to $20 \mathrm{~ms}$ ). We believe that these sawtooth-free periods (SFP) are due primarily to decreased current diffusion [and slowed peaking of $\mu(r)]$ arising from a reduced plasma resistivity. During SFP we observe that (1) the energy confinement time triples relative to normal sawtoothing discharges, and (2) the steady growth of the dominant $m=1$ modes normally observed between sawtooth crashes is absent. Also observed in SFP (and in some boronized discharges without SFP) are discrete bursts of $m=0$, toroidal mode number $n=1$ mode activity, each corresponding to a small increase in the toroidal flux.

Shown in Fig. 1 are data from a typical MST discharge in which sawteeth occur throughout. Each sawtooth crash causes a rapid increase in the overall toroidal magnetic flux [Fig. 1(a)], while enhancing the reversal of the toroidal magnetic field at the edge [Fig. 1(b)]. Increased particle transport at the crash induces plasma-wall interaction (e.g., sputtering), leading to injection of impurities into the plasma [Fig. 1(c)], an increase in the total radiated power [Fig. 1(d)], a decrease in temperature, and an increase in resistivity. (Note that impurity influx is sometimes observed just prior to a crash. This may be due in part to the magnetic fluctuations in the sawtooth rise phase.) Therefore, at each crash, the Ohmic input power [Fig. 1(e)] increases, and the stored thermal energy sometimes decreases. This leads to a temporary drop in the energy confinement time, $\tau_{E} \equiv$ (stored thermal energy)/ (Ohmic input power). The average $\tau_{E}$ in a sawtoothing discharge is about $1 \mathrm{~ms}$.

In Fig. 2 is shown a discharge with a long SFP. This discharge has a peak plasma current and line-averaged density similar to the discharge shown in Fig. 1. During the SFP, plasma-wall interaction is minimal [Figs. 2(c) and 2(d)], the plasma temperature is higher (than its usual between-crash value), and the resistivity is decreased, thereby leading to a decreased Ohmic input power [Fig. 2(e)]. The decreased plasma resistance also results in a flatter plasma current waveform [compare Fig. 2(f) to Fig. 1(f)].

At the plasma current shown in Figs. 1 and 2, the central electron temperature (measured with a Thomson scattering diagnostic) during SFP is $\sim 480 \mathrm{eV}$, compared to $\sim 275 \mathrm{eV}$ between sawteeth in normal discharges. This $\sim 75 \%$ increase 


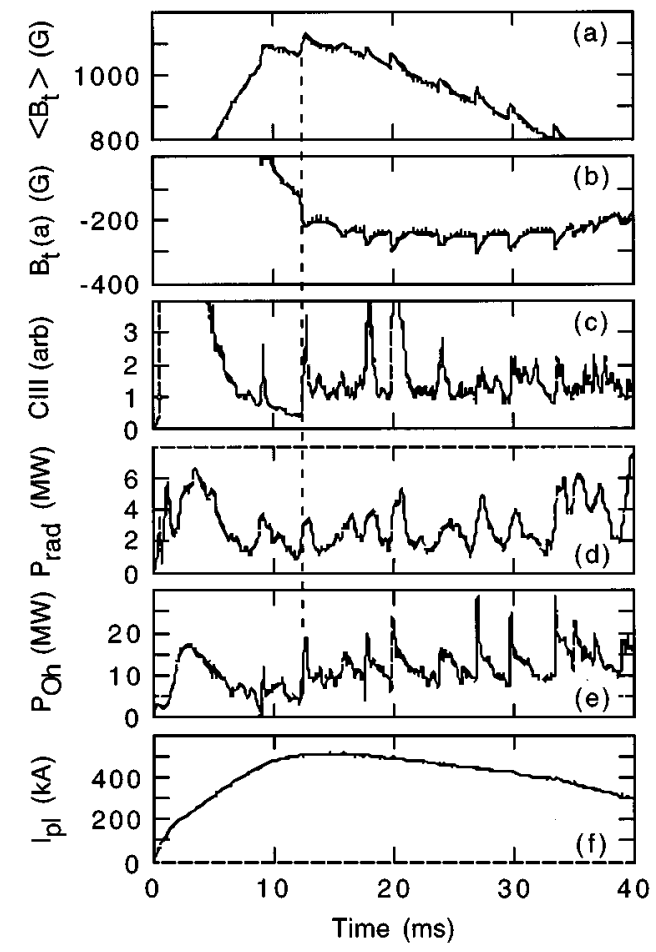

FIG. 1. Time variations of (a) the cross-section-averaged toroidal magnetic field, (b) the toroidal magnetic field at the plasma edge, (c) the CIII (464.74 $\mathrm{nm}$ ) intensity, (d) the total radiated power, (e) the Ohmic input power, and (f) the toroidal plasma current during a typical MST discharge. A sawtooth crash is indicated by a dashed line.

in temperature coupled with the lower Ohmic input power leads to a SFP $\tau_{E}$ of $\sim 3$ ms. Soft x-ray measurements indicate that the temperature and, thus, $\tau_{E}$ are sustained until the crash that ends the SFP.

The behavior of the dominant magnetic modes during SFP is quite different from that between sawtooth crashes in normal discharges. The dominant modes have $(m, n)=(1,6)$, $(1,7)$, and $(1,8)$ and are resonant at various radii in the plasma core. In Fig. 3(a) is shown the evolution of the $(1,6)$ during a complete sawtooth cycle in a boronized discharge. Its amplitude increases (in the rise phase) until the crash that begins at $\sim 13.4 \mathrm{~ms}$. In Fig. 3(d) is shown the evolution of the $(1,6)$ during a SFP later in the same discharge. Here, the mode maintains a nearly steady amplitude until the crash at the end of the SFP. This behavior is typical of the $(1,7),(1,8)$ and higher- $n m=1$ modes as well.

The behavior of the $(0,1)$ mode between sawtooth crashes depends on the condition of the plasma-facing wall. The $(0,1)$, like all $m=0$ modes, is resonant at the reversal radius (across which the toroidal magnetic field changes direction) near the plasma edge. In nonboronized discharges, the $(0,1)$ amplitude remains small between crashes. However, following boronization, the $(0,1)$ often exhibits quasiperiodic bursts, in both the sawtooth rise phase and SFP. The behavior of the $(0,1)$ in a complete sawtooth cycle is shown in Fig. 3(b), while its behavior in a SFP is shown in Fig. 3(e). In neither the sawtooth rise phase or SFP are these bursts correlated with changes in the $(1,6)$ or other $m=1$ mode amplitudes. At each sawtooth crash, however, the amplitudes of the $(0,1),(1,6)$, and most of the other detectable modes

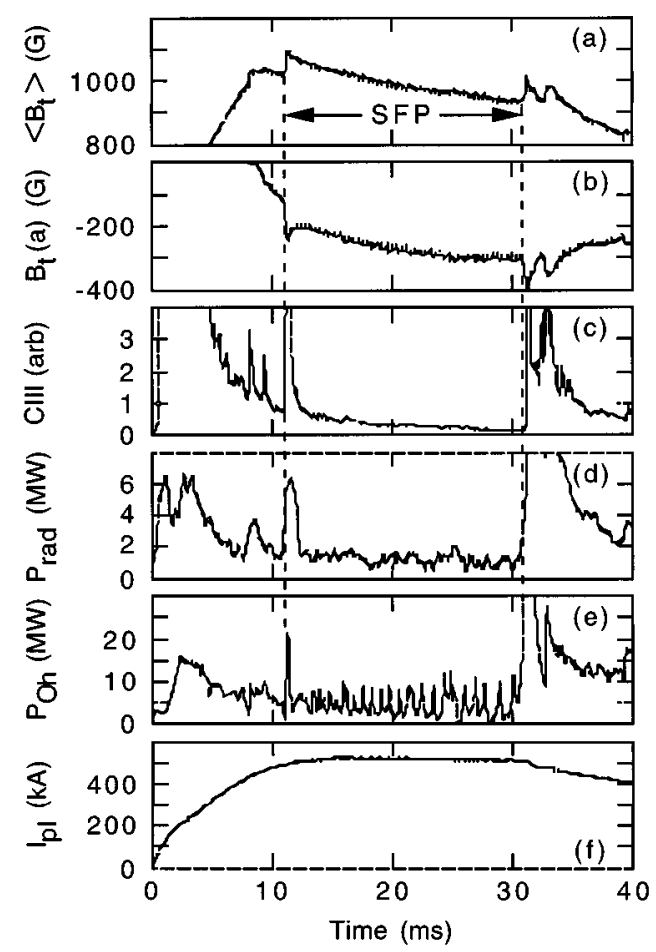

FIG. 2. Time variations of the plasma parameters in Fig. 1 during a discharge with a sawtooth-free period (SFP).

increase dramatically in unison. The effect is the same whether the crash follows a normal rise phase or a SFP.

Shown in Figs. 3(c) and 3(f) is the surface poloidal voltage, $V_{\theta}$. At each sawtooth crash, $V_{\theta}$ increases substantially, responding to the overall increase of toroidal flux. Such an increase, indicative of a dynamo event, is also observed at a smaller amplitude coincident with each burst of the $(0,1)$ mode. While each of these small-amplitude dynamo events causes a temporary increase in the Ohmic input power [seen clearly during the SFP shown in Fig. 2(e)], the events are benign compared to sawtooth crashes. One explanation for this, beyond the difference in amplitudes, is that the smallamplitude dynamo events appear to be localized to the plasma edge, in contrast to the sawtooth crashes which affect
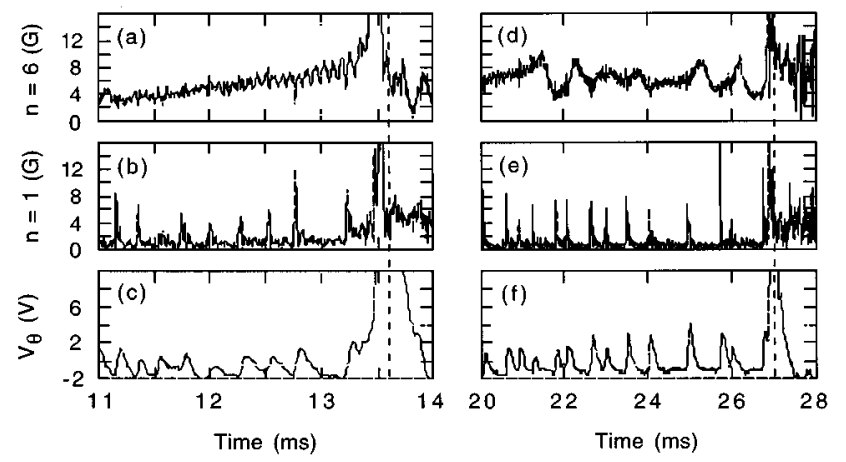

FIG. 3. Evolution of (a) the $(1,6)$ mode, (b) the $(0,1)$ mode, and (c) the surface poloidal voltage during a complete sawtooth cycle. Shown in (d)-(f) are these same signals during a sawtooth-free period later in the same discharge. Note the differing time scales. The dashed lines indicate the time at which the toroidal flux peaks during sawtooth crashes. Each crash begins with a rapid increase of the mode amplitudes. 


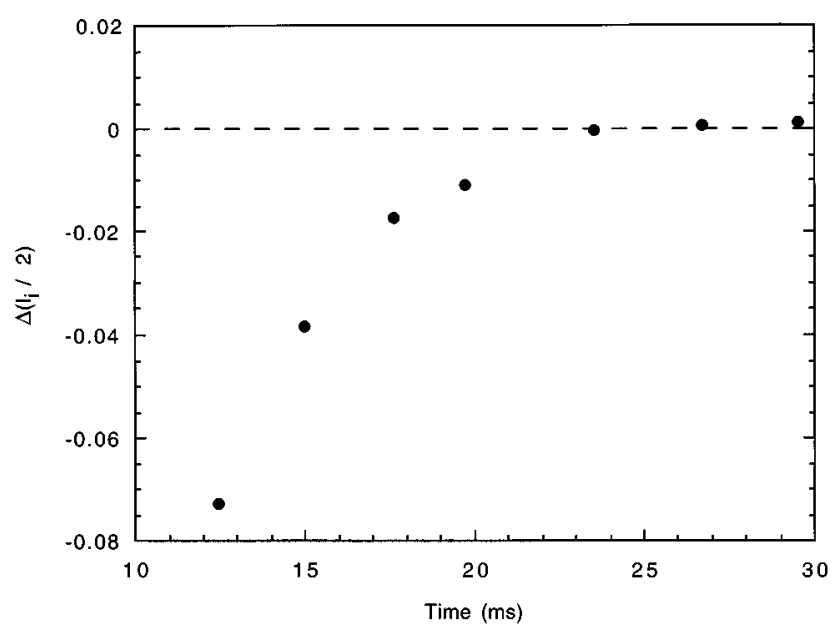

FIG. 4. $\Delta\left(l_{i} / 2\right)=\Lambda_{\mathrm{SFP}}\left(\right.$ Fig. 2) $-\Lambda_{\text {normal }}($ Fig. 1) -0.04 . The seven data points shown here correspond to $\Delta\left(l_{i} / 2\right)$ just before each crash in the normal discharge. The time window shown encompasses the sawtooth-free period.

the entire plasma. Edge localization of the small-amplitude dynamo events is suggested by the fact that they correlate well with the activity of the edge-resonant $(0,1)$ mode but not that of the core-resonant $m=1$ modes. In addition, observations of the electron density profile reveal that each event perturbs only the density near the plasma edge.

The cause of the small-amplitude $(0,1)$ bursts and the associated dynamo events is not yet known, but the fact that they are observed only after careful conditioning of the plasma-facing wall suggests a link to reduced plasma impurity content. Clearly, these events alone do not bring about SFP, but they may, like sawtooth crashes, contribute (in a small way) to the flattening of $\mu(r)$, thus helping to prolong the SFP.

The rate at which $\mu(r)$ peaks during SFP, relative to the normal sawtooth rise phase, can be estimated by measurement of the poloidal magnetic field distribution at the plasma boundary. This measurement allows calculation of the asymmetry factor, ${ }^{7} \Lambda \equiv\left(\beta_{\theta}+l_{i} / 2-1\right)$, where $\beta_{\theta}$ is the average poloidal beta, and $l_{i}$ is the plasma internal inductance. Large $l_{i}$ indicates a more peaked toroidal current density profile, which in the plasma core can indicate a peaked $\mu$ profile; $\Lambda$ was compared for the discharges shown in Figs. 1 and 2 during the time window encompassing the SFP of Fig. 2. Subtracting the average difference in $\beta_{\theta}$ between the two discharges, estimated to be $\sim 4 \%$, the remaining difference in $\Lambda$ is $\Delta\left(l_{i} / 2\right)$. The seven data points in Fig. 4 correspond to $\Delta\left(l_{i} / 2\right)$ just before each crash in the normal discharge. Early in time, the SFP $\mu(r)$ is flat compared to the peaked state reached before each sawtooth crash. Near the end of the SFP, however, $\mu(r)$ has peaked to the typical precrash level. Therefore, the delay in the onset of the sawtooth crash, which defines the SFP, results simply from slowed peaking of $\mu(r)$, apparently from a reduction of the plasma resistivity (and the current diffusion rate) following boronization. In other words, each SFP is roughly equivalent to an extended sawtooth rise phase. However, uncertainties in $\beta_{\theta}$ and $\Lambda$ do not preclude the possibility that the SFP $\mu(r)$ actually peaks beyond the normal precrash level. If resistivity alone accounts for the difference in the peaking rates, we can estimate the required change in $Z_{\text {eff }}$ (the mean ionic charge, not yet measured in the MST), given the known temperature difference between the two discharges. The average sawtooth rise time in the normal discharge is $\sim 3 \mathrm{~ms}$. Thus, the resistive diffusion time $\left(\propto Z_{\mathrm{eff}}^{-1} T_{e}^{3 / 2}\right)$ increases by a factor of $\sim 6$ in the SFP, requiring a factor of $\sim 2.6$ decrease in $Z_{\text {eff }}$, which is reasonable based on observations of the dominant impurities.

To summarize, the sawtooth-free period energy confinement time is triple the average value obtained during normal sawtoothing discharges. The usual steady growth of the dominant $m=1$ modes is absent, and the $(0,1)$ mode, following boronization, exhibits bursts associated with discrete dynamo events. The SFP confinement results are similar to those of experiments on the MST, also following boronization, where $\mu(r)$ was actively flattened by inductive means. ${ }^{8}$ Both experiments emphasize the need for steady-state control of both the current profile and the plasma impurity content in order to suppress sawteeth and ultimately suppress most of the residual magnetic fluctuations.

\section{ACKNOWLEDGMENTS}

The authors are grateful to C. R. Sovinec, N. Lanier, and the MST group.

This work was supported by the U.S. Department of Energy, including the Magnetic Fusion Science Fellowship Program.

${ }^{a)}$ Electronic mail: chapman@juno.physics.wisc.edu

${ }^{1}$ R. G. Watt and R. A. Nebel, Phys. Fluids 26, 1168 (1983).

${ }^{2}$ R. J. Hayden and B. Alper, Plasma Phys. Controlled Fusion 31, 193 (1989).

${ }^{3}$ S. Hokin, A. Almagri, S. Assadi, J. Beckstead, G. Chartas, N. Crocker, M. Cudzinovic, D. Den Hartog, R. Dexter, D. Holly, S. Prager, T. Rempel, J. Sarff, E. Scime, W. Shen, C. Spragins, C. Sprott, G. Starr, M. Stoneking, and C. Watts, Phys. Fluids B 3, 2241 (1991).

${ }^{4}$ R. N. Dexter, D. W. Kerst, T. W. Lovell, S. C. Prager, and J. C. Sprott, Fusion Technol. 19, 131 (1991).

${ }^{5}$ J. A. Beckstead, Ph.D. thesis, University of Wisconsin, Madison, Wiscon$\sin , 1990$.

${ }^{6}$ D. J. Den Hartog, M. Cekic, G. Fiksel, S. A. Hokin, R. D. Kendrick, S. C. Prager, and M. R. Stoneking, J. Nucl. Mater. 200, 177 (1993); D. J. Den Hartog and R. D. Kendrick, ibid. 220-222, 631 (1995).

${ }^{7}$ V. S. Mukhovatov and V. D. Shafranov, Nucl. Fusion 11, 605 (1971).

${ }^{8}$ J. S. Sarff, S. A. Hokin, H. Ji, S. C. Prager, and C. R. Sovinec, Phys. Rev. Lett. 72, 3670 (1994). 